INTENSIV BETREUTE FRÜHCHEN

\title{
Der Schmerz wirkt bis ins Jugendalter nach
}

- Kinder, die vor der 32. Schwangerschaftswoche auf die Welt kommen und in ihren ersten Tagen schmerzhaften Prozeduren ausgesetzt werden, sind noch lange besonders schmerzempfindlich.

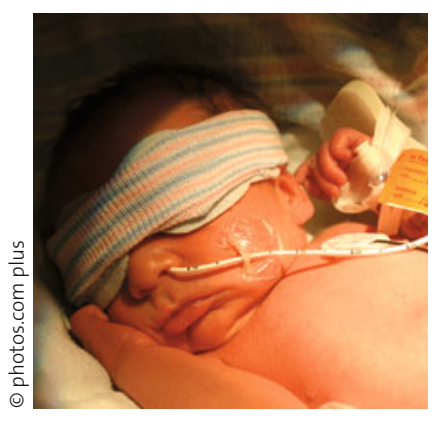

Frühchen brauchen eine sorgfältige Schmerzbehandlung.
Psychologen der Universität Gießen wiesen mithilfe funktioneller Kernspintomografie bis zum Alter von 16 Jahren eine stärkere Gehirnreaktion nach einem schmerzhaften Hitzereiz nach als bei Kindern ohne frühen Krankenhausaufenthalt. Auch der sonst übliche Gewöhnungseffekt nach mehrfachen Schmerzreizen trat nicht ein.

ST :

Pain 2010;150:257-267;

doi:10.1016/j.pain.2010.04.004

\section{AUGEN AUF BEIM AUTOFAHREN}

\section{Neues Warnsystem gegen Sekundenschlaf}

— Eine Minikamera im Armaturenbrett des Autos soll künftig verhindern, dass der Fahrer einnickt. Registriert das System, dass das Auge länger als die variabel einstellbare Zeit, z.B. eine Sekunde, geschlossen ist, schlägt es Alarm. Der Hintergrund: Jeder vierte Unfall auf der Autobahn mit Todesfolge geht laut Deutschem Verkehrssicherheitsrat auf einen Sekundenschlaf zurück. Das neue Assistenzsystem, das vom Fraunhofer-Institut für Digitale Medientechnologie entwickelt wurde, wird auf der VISION in Stuttgart vom 9.-11. November vorgestellt. Es verfügt über mindestens zwei Kameras und kann so die räumliche Lage der Pupille und die Blickrichtung registrieren.

Quelle: Pressemeldung der Fraunhofer-Gesellschaft

\section{VIELE KREBSTHERAPIEN GREIFEN DAS HERZ AN}

\section{Kardio-Onkologie als neue Disziplin}

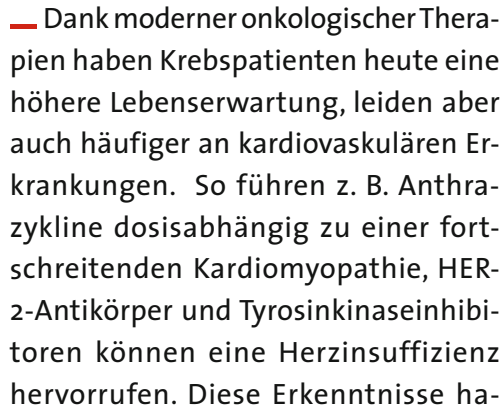

ben eine neue Disziplin auf den Plan gerufen: die Kardio-Onkologie. Sie soll auch dafür sorgen, dass Herzpatienten, die an Krebs erkranken, nicht von kardiologischer Seite vernachlässigt werden bzw. dass Krebspatienten trotz einer Herzerkrankung ausreichend aggressive onkologische Therapien erhalten.

BS theheart.org, 8. Oktober 2010

\section{Risikocheck beim Hausarzt}

Im Kampf gegen Herzinfarkt und plötzlichen Herztod kommt die Primärprävention hierzulande immer noch viel zu kurz. Während Patienten mit einer manifesten KHK im Großen und Ganzen recht gut versorgt sind, gibt es bei der Vorsorge und bei der Früherkennung von Herzerkrankungen noch immer Lücken. Die Siemens-Betriebskrankenkasse (SBK) in München will das ändern. Bereits 2006 hat die Kasse für ihre Versicherten ein spezielles Vorsorge- und Früherkennungsprogramm aufgelegt. In den kommenden Monaten wird die SBK dafür die Werbetrommel besonders intensiv rühren.

Mit den Themenwochen „Starkes Herz“, die bis zum März 2011 laufen, will die SBK ihre Versicherten für einen herzgesunden Lebensstil motivieren. Dazu gibt es eine großangelegte Informationskampagne mit Vortragsveranstaltungen und Kantinenaktionen, mit Aufklebern, Broschüren und Flyern, in denen auch für eine kostenlose Vorsorgeuntersuchung beim Hausarzt geworben wird.

Mit dem SBK KardioPro Risikochek, der sich an gesunde Versicherte ab 45 Jahren wendet, wird das individuelle Risiko bestimmt, in den nächsten zehn Jahren einen Herzinfarkt zu erleiden. Die geeigneten Maßnahmen und Behandlungen können so rechtzeitig eingeleitet werden. Auf diese Weise soll der Eintritt eines Herzinfarktes

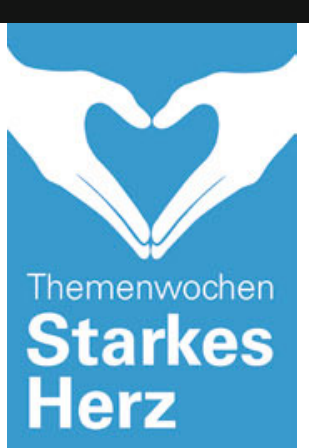

oder einer chronischen Herzerkrankung vermieden oder zumindest verzögert werden.

Dass durch einen solchen Risikochek zahlreiche bislang nicht erkannte KHKPatienten entdeckt werden, zeigt eine Studie der SBK mit mehr als 10 ooo Versicherten ohne bekannte KHK: Nahezu jeder fünfte Versicherte hatte ein mittleres und $11 \%$ hatten sogar ein hohes kardiovaskuläres Risiko.

Die begleitenden Unterlagen der SBK können angefordert werden bei der SBK-Zentrale, Frau Susanne Weber unter 07321/303-143 oder susanne. weber@sbk.org. 